\title{
Correction to: Associations Between Child and Administrator Race and Suspension and Expulsion Rates in Community Childcare Programs
}

\author{
Keri Giordano ${ }^{1} \mathbb{D} \cdot$ Victoria L. Interra ${ }^{1} \cdot$ Giuliana C. Stillo $^{1} \cdot$ Angel T. Mims $^{1} \cdot$ Jennifer Block-Lerner ${ }^{1}$
}

Published online: 23 March 2020

(c) Springer Nature B.V. 2020

\section{Correction to: Early Childhood Education Journal https://doi.org/10.1007/s10643-020-01049-1}

The original version of this article unfortunately contained a mistake in Table 1 . The correct Table 1 is given below:

Publisher's Note Springer Nature remains neutral with regard to jurisdictional claims in published maps and institutional affiliations.
Table 1 Student race as a predictor of disproportionate suspension rates

\begin{tabular}{lll}
\hline Race & $\begin{array}{l}\text { Number of children } \\
\text { suspended }\end{array}$ & Risk ratio \\
\hline White & 74 & 1.15 \\
Black/African American & 42 & 3.43 \\
Hispanic/Latino/Spanish & 16 & .71 \\
Multi-racial/multi-ethnic & $13^{\mathrm{a}}$ & 1.85 \\
Asian/Asian American & $8^{\mathrm{a}}$ & .72 \\
Middle Eastern/North African & $5^{\mathrm{a}}$ & 1.45 \\
Other race or origin & $3^{\mathrm{a}}$ & .88 \\
Unknown & $10^{\mathrm{a}}$ & .20 \\
Native Hawaiian/Pacific Islander & $0^{\mathrm{a}}$ & 0 \\
American Indian/Alaskan native & $0^{\mathrm{a}}$ & 0
\end{tabular}

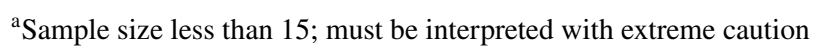

The original article can be found online at https://doi.org/10.1007/ s10643-020-01049-1.

Keri Giordano

KGiordan@kean.edu

1 Kean University, 1000 Morris Avenue, Union, NJ 07083, USA 